\title{
EQUTVALENCES GENERATED BY FAMILIES OF BOREL SETS
}

\author{
JOHN P. BURGESS ${ }^{1}$
}

\begin{abstract}
The equivalence relation on the reals generated by a family of $\boldsymbol{N}_{\alpha}$ Borel sets has either $<\boldsymbol{N}_{\alpha}$ or else exactly $2^{\boldsymbol{N}_{0}}$ equivalence classes.
\end{abstract}

As is usual in modern set theory, we identify an ordinal with the set of its predecessors, and a cardinal with the first ordinal of that cardinality. Thus $2=\{0,1\}, \omega=\{0,1,2, \ldots\}$; while $\boldsymbol{x}_{0}=\omega, \boldsymbol{\kappa}_{1}=$ the first uncountable ordinal $\omega_{1}$, etc. If $\alpha, \beta$ are ordinals,

$$
{ }^{\beta} \alpha=\{f: f \text { is a function } \& \operatorname{dom} f=\beta \& \text { range } f \subseteq \alpha\} ;
$$

while

$$
{ }^{\beta} \alpha=\bigcup_{\gamma<\beta}{ }^{\gamma} \alpha .
$$

If $f \in{ }^{\beta} \alpha$ and $\gamma<\beta$, then $f \mid \gamma$ is the restriction of $f$ to $\gamma$; while if $\delta<\alpha, f * \delta$ is the element $g$ of ${ }^{(\beta+1)} \alpha$ with $g \mid \beta=f$ and $g(\beta)=\delta$.

Let $X$ be an uncountable Polish space (separable topological space admitting a complete metric), e.g. the reals. A family $\delta$ of subsets of $X$ generates an equivalence relation $E(\mathcal{S})$ on $X$ defined by

$$
x E(\delta) y \leftrightarrow \forall S \in \delta(x \in S \leftrightarrow y \in S) .
$$

Let $\kappa$ be an infinite cardinal. A subset $S \subseteq X$ is called $\kappa$-Souslin if $S$ can be represented in the form

$$
S=\bigcup_{f \in \omega_{\kappa}} \bigcap_{n \in \omega} C_{f \mid n},
$$

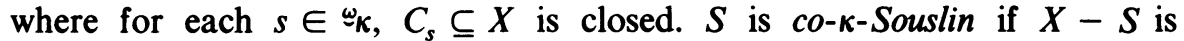
$\kappa$-Souslin, and bi-k-Souslin if both $\kappa$-Souslin and co- $\kappa$-Souslin. Thus the $\omega$-Souslin sets are just the analytic $\left(\Sigma_{1}^{1}\right)$ sets; the co- $\omega$-Souslin sets are the $C A\left(\Pi_{1}^{\prime}\right)$ sets; and by a classical theorem of Souslin (see [3]) the bi- $\omega$-Souslin sets are the Borel sets.

An equivalence relation $E$ on $X$ is said to have perfectly many classes if there is a perfect (closed, dense-in-itself) $P \subseteq X$ such that no two (distinct) elements of $P$ are $E$-equivalent. Since any perfect subset of $X$ has cardinality $2^{\kappa_{0}}$, this implies $E$ has $2^{\kappa_{0}}$ classes. Note that if $\mathcal{S}, \mathcal{T}$ are families of subsets of

Received by the editors March 25, 1977.

AMS (MOS) subject classifications (1970). Primary 04A15.

${ }^{1}$ Research supported in part by the National Science Foundation MCS76-10224. 
$X$ with $\mathscr{T} \subseteq \mathcal{S}$, then $E(\mathcal{S}) \subseteq E(\mathcal{T})$ (as subsets of $X^{2}$ ), and, hence, the number of $E(\delta)$ classes can be no less than the number of $E(\mathscr{T})$ classes, and the former has perfectly many classes if the latter does.

THEOREM. Let $X$ be an uncountable Polish space, $\kappa$ an infinite cardinal, $\delta$ a family of $\kappa$ many bi- $\kappa$-Souslin subsets of $X$. Then if the equivalence relation $E(\mathcal{S})$ generated by $\mathcal{S}$ has more than $\kappa$ equivalence classes, there exists a countable $\mathscr{T} \subseteq \mathcal{S}$ such that $E(\mathcal{T})$ has perfectly many classes.

Proof. Fix a complete metric $\rho$ on $X$ compatible with its topology. Enumerate $\mathcal{S}=\left\{S^{\alpha}: \alpha<\kappa\right\}$. For each $\alpha<\kappa$ fix families ${ }^{i} C_{s}^{\alpha}$ of closed subsets of $X$ for $i=0,1$ and $s \in \omega_{\kappa}$, such that:

$$
S^{\alpha}=\bigcup_{f \in \omega_{\kappa}} \bigcap_{n \in \omega}{ }^{0} C_{f \mid n}^{\alpha}, \quad X-S^{\alpha}=\bigcup_{f \in \omega_{\kappa}} \bigcap_{n \in \omega}{ }^{1} C_{f \mid n}^{\alpha} .
$$

We may choose these families to be nested, so $s \subseteq t$ implies ${ }^{i} C_{t}^{\alpha} \subseteq{ }^{i} C_{s}^{\alpha}$; and we may choose them so that for $n \in \omega$ and $s \in{ }^{n} \kappa$, the $\rho$-diameter of ${ }^{i} C_{s}^{\alpha}$ is less than $2^{-n}$. For $\alpha<\kappa, i=0,1$, and $s \in{ }^{\omega}{ }_{\kappa}$, set

$$
{ }^{i} S_{s}^{\alpha}=\bigcup_{\substack{f \in \omega_{K} \\ s \subseteq f}} \bigcap_{n \in \omega}{ }^{i} C_{f \mid n}^{\alpha} \subseteq{ }^{i} C_{s}^{\alpha} .
$$

Assume $E(\delta)$ has $>\kappa$ classes, and let $Z \subseteq X$ be a set of $\kappa^{+}$pairwise $E(\delta)$-inequivalent elements.

We will define for every $l \in \omega, \sigma \in{ }^{\prime} 2$, an ordinal $\alpha(\sigma)<\kappa$ and elements $s(\sigma, k)$ of ${ }^{\prime} k$ for $k \leqslant l$, so that setting

$$
T_{\sigma}=\bigcap_{k<l}{ }^{\sigma(k)} S_{s(\sigma, k)}^{\alpha(\sigma \mid k)} \subseteq \bigcap_{k<l}{ }^{\sigma(k)} C_{s(\sigma, k)}^{\alpha(\sigma) k)},
$$

we have $\operatorname{card}\left(Z \cap T_{\sigma}\right)=\kappa^{+}$. We will also arrange matters so that $\sigma \subseteq \tau$ implies $s(\sigma, k) \subseteq s(\tau, k)$ for all relevant $k$. We proceed by induction. Suppose then that $l \in \omega, \sigma \in{ }^{\prime} 2$, and suppose that for all $k<l, \alpha(\sigma \mid k)$ and $s(\sigma, k)$ have been defined and satisfy the conditions above.

In particular, $\operatorname{card}\left(Z \cap T_{\sigma}\right)=\kappa^{+}$. We claim this assumption implies that there exists an $\alpha<\kappa$ such that both $Z \cap T_{\sigma} \cap S^{\alpha}$ and $\left(Z \cap T_{\sigma}\right)-S^{\alpha}$ have cardinality $\kappa^{+}$. For suppose the opposite, and setting, for each $\alpha<\kappa$, $M^{\alpha}=$ whichever of $Z \cap T_{\sigma} \cap S^{\alpha}$ or $\left(Z \cap T_{\sigma}\right)-S^{\alpha}$ has cardinality $\leqslant \kappa$, we would find that all elements of $Z-\cup_{\alpha<\kappa} M^{\alpha}$ would be $E(\delta)$ )-equivalent, hence that there could be only one such element, hence that card $Z=\kappa$, a contradiction! Let $\alpha(\sigma)$ be the least $\alpha$ with $\operatorname{card}\left(Z \cap T_{\sigma} \cap S^{\alpha}\right)=\operatorname{card}((Z \cap$ $\left.\left.T_{\sigma}\right)-S^{\alpha}\right)=\kappa^{+}$. Now $Z \cap T_{\sigma} \cap S^{\alpha(\sigma)}$ is contained in

$$
\bigcap_{k<l}{ }^{\sigma(k)} S_{s(\sigma, k)}^{\alpha(\sigma \mid k)} \cap S^{\alpha(\sigma)}=\bigcap_{k<l} \bigcup_{\nu<\kappa}{ }^{\sigma(k)} S_{s(\sigma, k) * \nu}^{\alpha(\sigma \mid k)} \cap \bigcup_{s \in(1+1)_{k}}{ }^{0} S_{s}^{\alpha(\sigma)} .
$$

So there exist $\nu_{0}, \nu_{1}, \ldots, \nu_{(l-1)}$ and $s$ such that setting $s(\sigma * 0, k)=$ $s(\sigma, k) * \nu_{k}$ for $k<l$, and $s(\sigma * 0, l)=s$, and defining $T_{\sigma} .0$ as per (1) above, 
we still have $\operatorname{card}\left(Z \cap T_{\sigma * 0}\right)=\kappa^{+}$. The $s(\sigma * 1, k)$ for $k \leqslant l$ are similarly defined.

For $g \in{ }^{\omega} \omega,\left\{\bar{T}_{g \mid n}: n \in \omega\right\}$ forms a nested sequence of nonempty closed sets with $\rho$-diameters converging to 0 . Hence this family intersects in a point $x_{g} \in X$. If $g(m)=0$, then $x_{g}$ belongs to

$$
\bigcap_{n>m}{ }^{0} C_{s(g \mid n, m)}^{\alpha(g \mid m)} \subseteq S^{\alpha(g \mid m)}
$$

Similarly, if $g(m)=1$, then $x_{g} \notin S^{\alpha(g \mid m)}$. Thus if $g, h$ are two (distinct) elements of ${ }^{\omega} \omega, x_{g}, x_{h}$ are $E(\mathcal{S})$-inequivalent, and incidentally $x_{g} \neq x_{h}$. Thus $A=\cup_{g \in \omega_{\omega}} \cap{ }_{n \in \omega} \bar{T}_{g \mid n}=\left\{x_{g}: g \in{ }^{\omega} \omega\right\}$ is an uncountable analytic set, and hence contains a perfect subset $P$. Moreover, setting $\mathcal{T}=\left\{S^{\alpha(\sigma)}: \sigma \in{ }^{\omega} 2\right\}$, any two elements of $P$ are $E(\mathcal{T})$-inequivalent, proving the theorem.

Corollary 1. Let $X$ be a Polish space, $\kappa$ an infinite cardinal. Then any equivalence relation on $X$ which is an intersection of $\kappa C A$ equivalences has either $\leqslant \kappa$ or else perfectly many equivalence classes.

Proof. We use a deep theorem of Silver [4]: Any $C A\left(\Pi_{1}^{1}\right)$ equivalence relation on a Polish space $X$ has either countably many or else perfectly many equivalence classes. Now let $E$ be an equivalence on a Polish space $X$ of form $\cap_{\alpha<\kappa} E_{\alpha}$ where the $E_{\alpha}$ are $C A$ equivalences. If any $E_{\alpha}$ has perfectly many classes, so does $E$. If each $E_{\alpha}$ has only countably many classes $\left\{S_{\alpha, n}\right.$ : $\left.n<N_{\alpha}\right\}, N_{\alpha} \leqslant \omega$, then each of these $S_{\alpha, n}$ is both $C A$ (since $E_{\alpha}$ is $C A$ ) and analytic (being the complement of $\cup_{m \neq n} S_{\alpha, m}$ ) and hence is Borel. Thus in this case $E=E(\mathcal{S})$ where $\mathcal{S}=\left\{S_{\alpha, n}: \alpha<\kappa, n<N_{\alpha}\right\}$ is a family of $\kappa$ Borel sets. Thus any intersection of $\kappa C A$ equivalences either has perfectly many classes or else is generated by a family of $\kappa$ Borel sets. Corollary 1 is immediate. This corollary answers a question of $\mathrm{J}$. Steel.

The referee has informed us that V. Harnik and M. Makkai [5] have obtained Corollary 1 (for $X=$ Baire space) by a model-theoretic argument. The Theorem has somewhat more scope than this corollary, implying e.g. that if $\delta$ is a family of $\aleph_{1}$ analytic sets, $E(\delta)$ has $\leqslant \aleph_{1}$ or perfectly many classes.

COROllary 2. Any analytic equivalence relation on a Polish space $X$ has either $\leqslant \omega_{1}$ or else perfectly many classes.

Proof. Elsewhere [2] we have shown: Any analytic equivalence relation on a Polish space $X$ is an intersection of $\omega_{1}$ Borel equivalences. Corollary 2 is then immediate. Actually in [2] we establish more: A CPCA( $\left.\mathbf{\Pi}_{2}^{1}\right)$ equivalence of the special form $x E y \leftrightarrow \forall z \in X(x, y, z) \in D$, where $D \subseteq X^{3}$ is analytic, and for each fixed $z,\{(x, y):(x, y, z) \in D\}$ is an equivalence relation, is an intersection of $\omega_{1} C A$ equivalences. So the cardinal estimates on the number of classes in Corollary 2 apply to such special $C P C A$ equivalences, too. Corollary 2 was the main result of our thesis [1]. It answers a question of $\mathrm{H}$. Friedman. 


\section{BIBLIOGRAPHY}

1. J. P. Burgess, Infinitary languages and descriptive set theory, Doctoral Dissertation, Univ. of California, Berkeley, Calif., 1974.

2. __ A reflection phenomenon in descriptive set theory, Fund. Math. (to appear).

3. K. Kuratowski, Topology, Vol. 1, Academic Press, New York, 1966.

4. J. H. Silver, $\mathbf{I}_{1}^{\prime}$ equivalence relations (to appear).

5. V. Harnik and M. Makkai, $A$ tree argument in infinitary model theory, Proc. Amer. Math. Soc. 67 (1977), 309-314.

Department of Philosophy, Princeton University, Princeton, New Jersey 08540 